\title{
POLARIZATION OBSERVATIONS OF SUPERNOVA REMNANTS
}

\author{
D. K. MILNE \\ Division of Radiophysics, CSIRO, Sydney, Australia \\ and \\ JOHN R. DICKEL \\ University of Illinois Observatory, Urbana, Ill., U.S.A.
}

\begin{abstract}
Polarization and total power at $5000 \mathrm{MHz}$ and $2700 \mathrm{MHz}$ have been obtained for 30 supernova remnants (SNR) using the 64-m radio telescope at Parkes. This large sample includes a range of SNR from the young bright objects (such as Kepler's SNR) through the old faint sources such as MSH 14-63. Among the old remnants, the individual SNR have very varied properties, but several general conclusions emerge from our study. (1) In many cases, the polarization of the galactic background is as strong as that of the SNR and can vary significantly over the angular extent of the SNR. (2) The Faraday rotation is generally small and varies quite uniformly across the source. (3) The magnetic field patterns, although associated with the SNR, do not show any particularly characteristic pattern or relations to the totalpower structure of the source.
\end{abstract}

\section{Introduction}

We have obtained 6- and 11-cm polarization and total power maps of 30 supernova remnants (SNR) using the 64-m radio telescope at Parkes. This large sample includes a range of SNR from the young bright objects (such as Kepler's SNR) through the old faint sources such as MSH 14-63. Among the old remnants, the individual SNR have very varied properties but several general conclusions emerge from our study: (i) In many cases, the polarization of the galactic background is as strong as that of the SNR and can vary significantly over the angular extent of the SNR; (ii) the Faraday rotation is generally small and varies quite uniformly across the source; and (iii) the magnetic field patterns, although associated with the SNR, do not show any particularly characteristic pattern or relations to the total power structure of the source. These points will be described below.

\section{Equipment and Observations}

At $2700 \mathrm{MHz}$ we used a two-channel parametric correlation receiver, giving a system noise temperature of $100 \mathrm{~K}$ over a $400-\mathrm{MHz}$ band, and at $5000 \mathrm{MHz}$ a switched parametric receiver with an $80-\mathrm{K}$ noise temperature and $500-\mathrm{MHz}$ bandwidth was used. Both of these receivers compared the signals from probes of opposite polarization in circular waveguide feed horns. The beamwidths were $8.4^{\prime}$ and $4.4^{\prime}$ at 2700 and $5000 \mathrm{MHz}$, respectively.

The observing procedures have been described in detail by Milne (1972) and consisted, briefly, of scans in declination spaced at approximate half-beamwidth intervals in right ascension. At each right ascension a total of four scans were made, at position angles separated by $45^{\circ}$. The polarization $\mathbf{E}$ vectors were then obtained 
by combining these scans in the usual way. At $2700 \mathrm{MHz}$ two instrumental effects were recognized and removed;(i) a 'gain' effect yielding a spurious polarization vector always at the same feed angle and (ii) a spurious polarization proportional to the total power intensity gradient and directed along this gradient. No significant instrumental effects were detected at $5000 \mathrm{MHz}$.

\section{Results}

\section{(a) BACKGROUND POLARIZATION}

A good example of the extremely large background contribution which can occur in the direction of some sources is seen in Figure 1. This shows the polarization $\mathbf{E}$

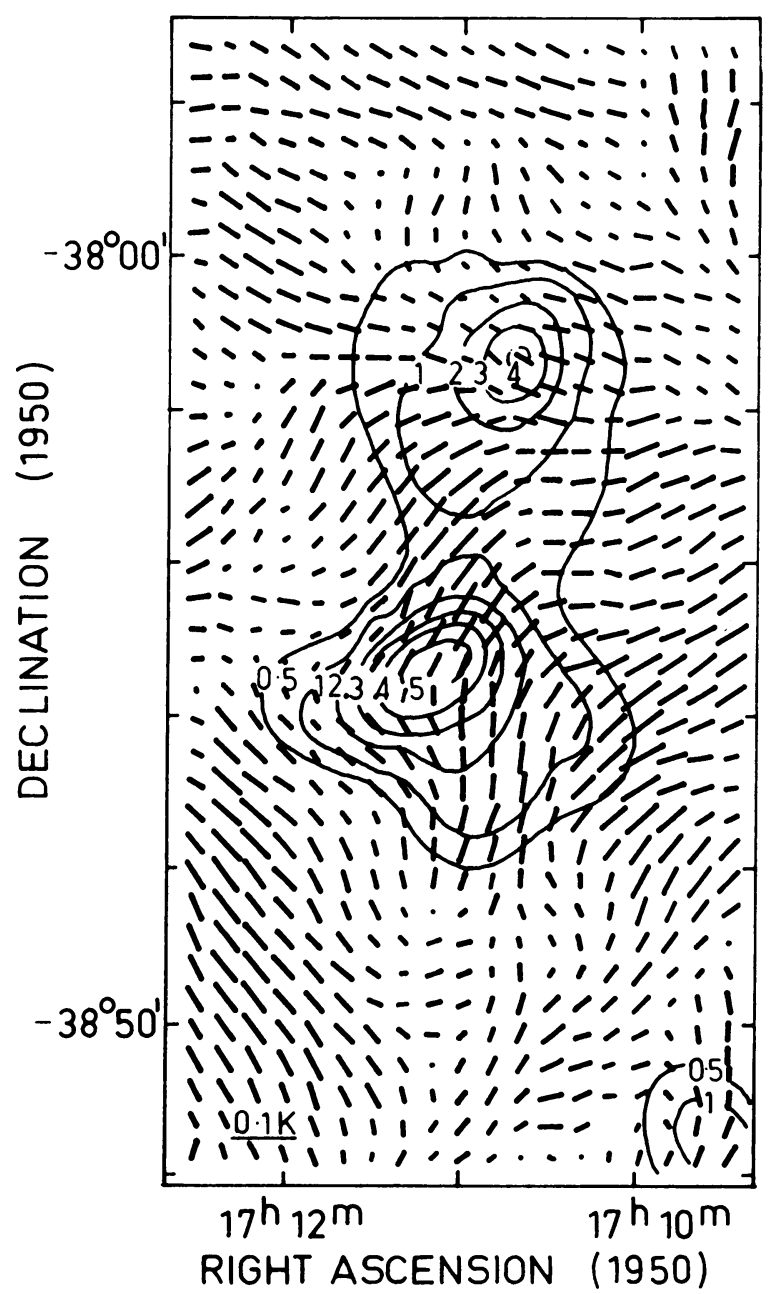

Fig. 1. The high level of background polarization is seen here in the $5000-\mathrm{MHz}$ E vectors around CTB $37 A$ and $B$. The total power contour unit is $1 \mathrm{~K}$ in brightness temperature in all of these figures. 
vectors at $6 \mathrm{~cm}$ in the field of CTB $37 A$ and $B$, two separate SNRs (G348.5+0.1 and G348.7+0.3). It is virtually impossible to distinguish where the SNRs should be from this figure; the polarization and the SNRs bear no relation. Presumably the strong galactic magnetic fields and perhaps intervening Faraday rotation have masked the source polarization. The source MSH $11-61 A(\mathrm{G} 290.1-0.8)$ is an example of an SNR whose polarization clearly stands out above the background, although the latter is still significantly polarized (Figure 2). It can also be seen that the background polarization changes significantly across the $15^{\prime}$ subtended by the SNR. In this case the H II region MSH $11-61 B$ just to the west of the SNR shows negligible polarization, as would be expected for such an object. A final example of the background polarizations is shown in Figure 3a. Although the SNR MSH 14-63 (G315.4-2.3) is faint its polarization can be seen to just stand out above the noise as well as the background. Even without the total power contours the general position of the source can be picked out from the map of the magnetic fields alone in Figure $3 \mathrm{~b}$. In this case we plot the directions of the projected magnetic field, i.e., orthogonal to the observed $\mathbf{E}$ vectors after removal of the Faraday rotation.

(b) FARADAY ROTATION

The Faraday rotation was evaluated from the two-frequency data after convolution to the same effective resolution for both; the lowest value of $|R M|$ generally was

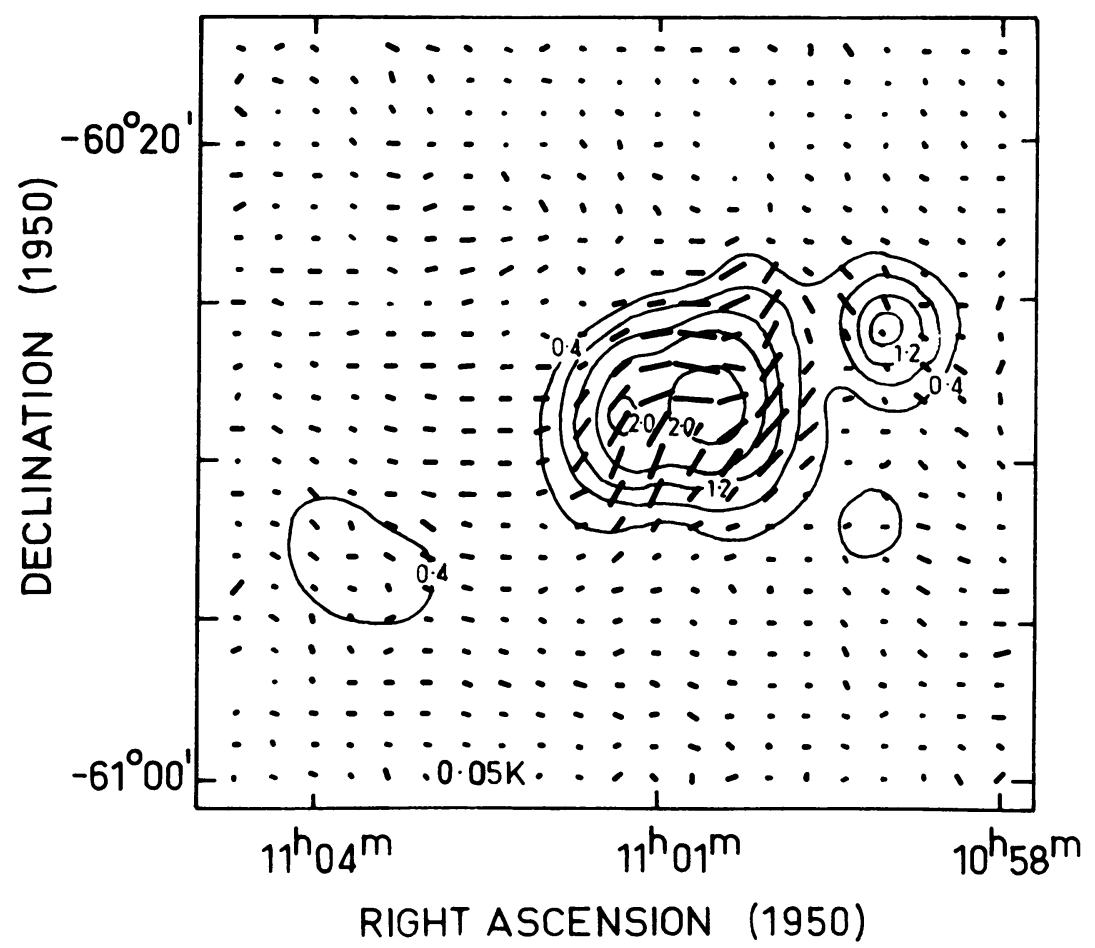

Fig. 2. $5000-\mathrm{MHz}$ polarization $\mathbf{E}$ vectors and total power contours for $\mathrm{MSH} 11-61 \mathrm{~A}$ and $B$. 


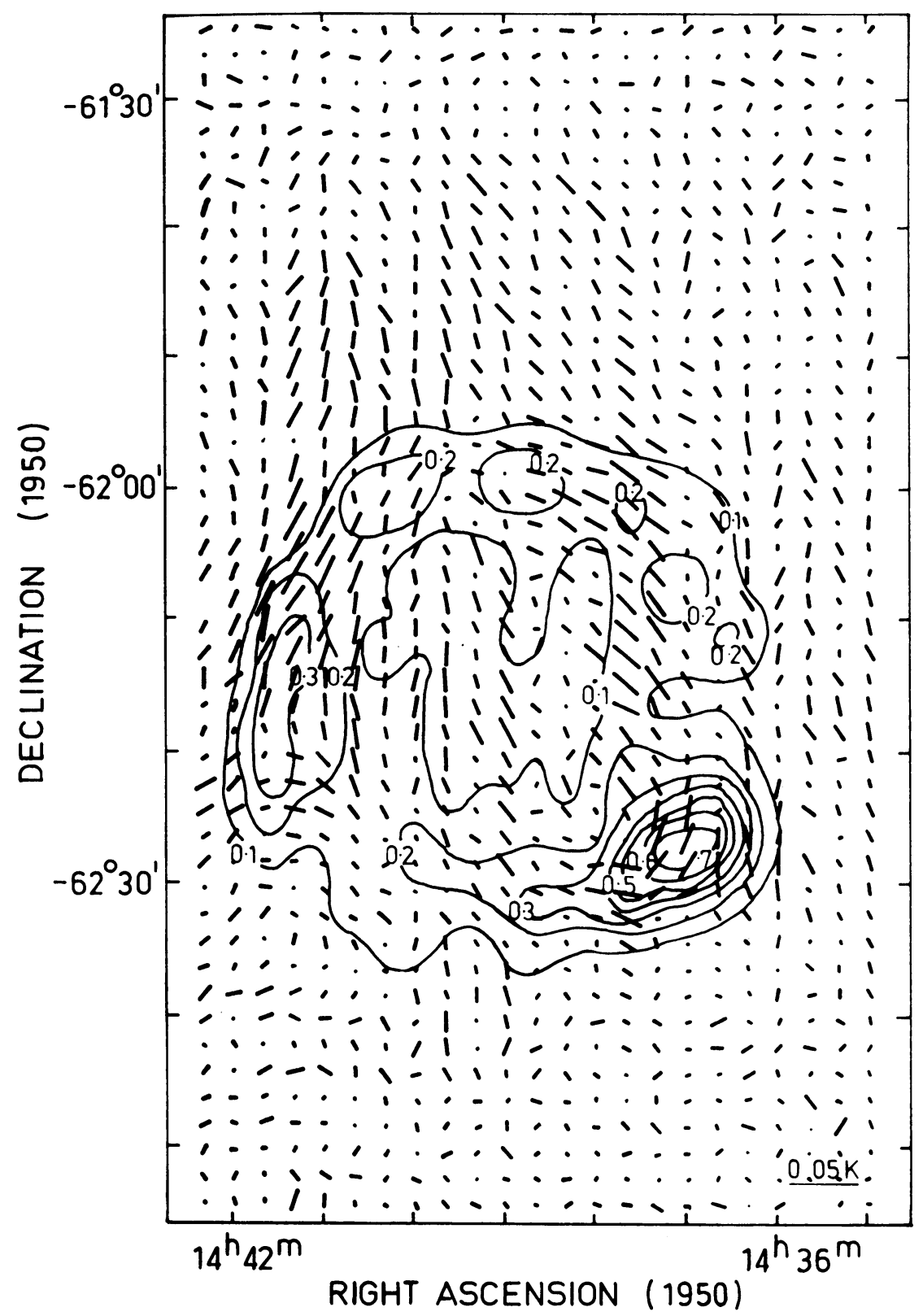

Fig. 3a. $\quad 5000-\mathrm{MHz}$ polarization $\mathbf{E}$ vectors for $\mathrm{MSH} 14-63$. 


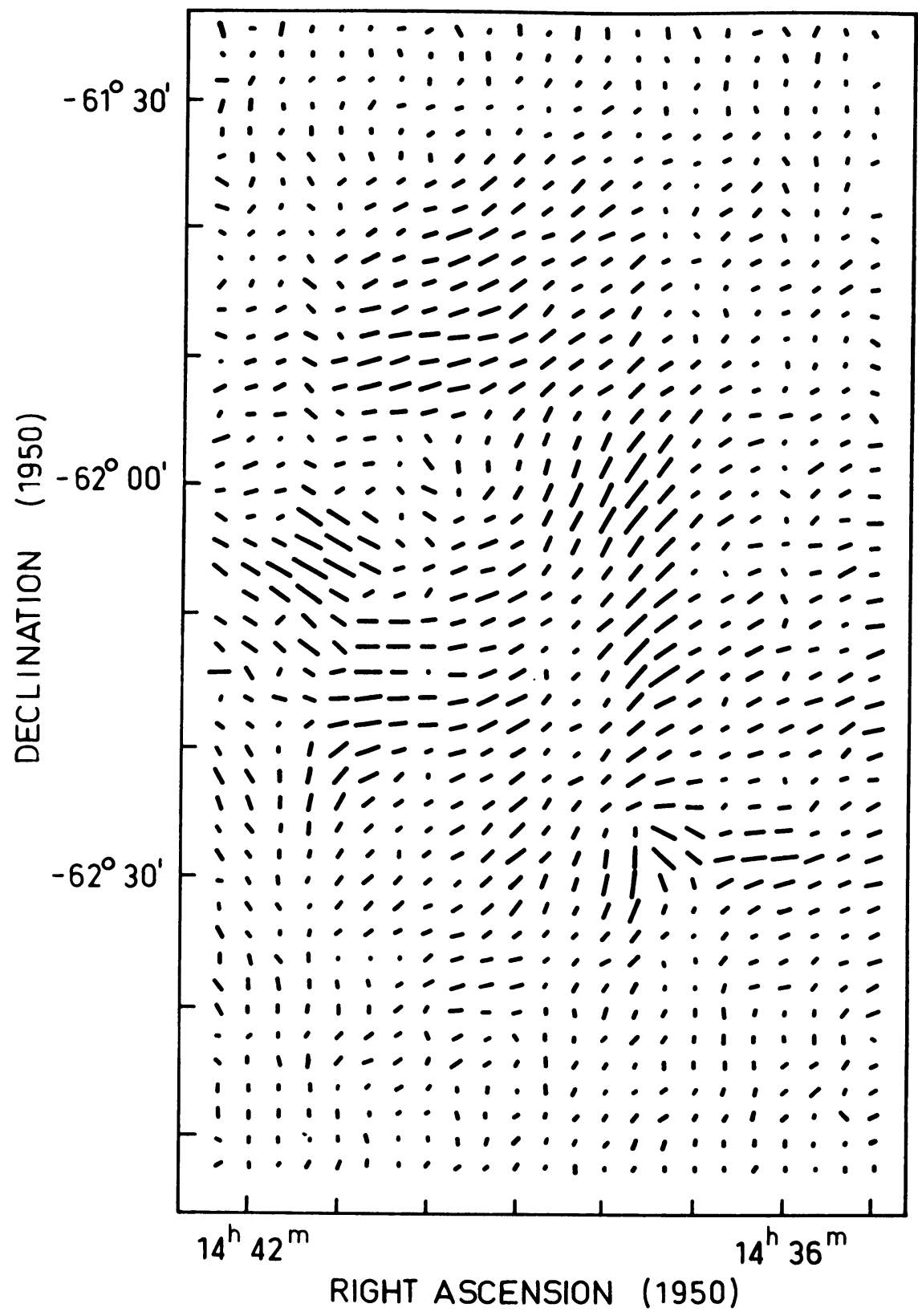

Fig. 3b. Directions of magnetic field for MSH 14-63. 


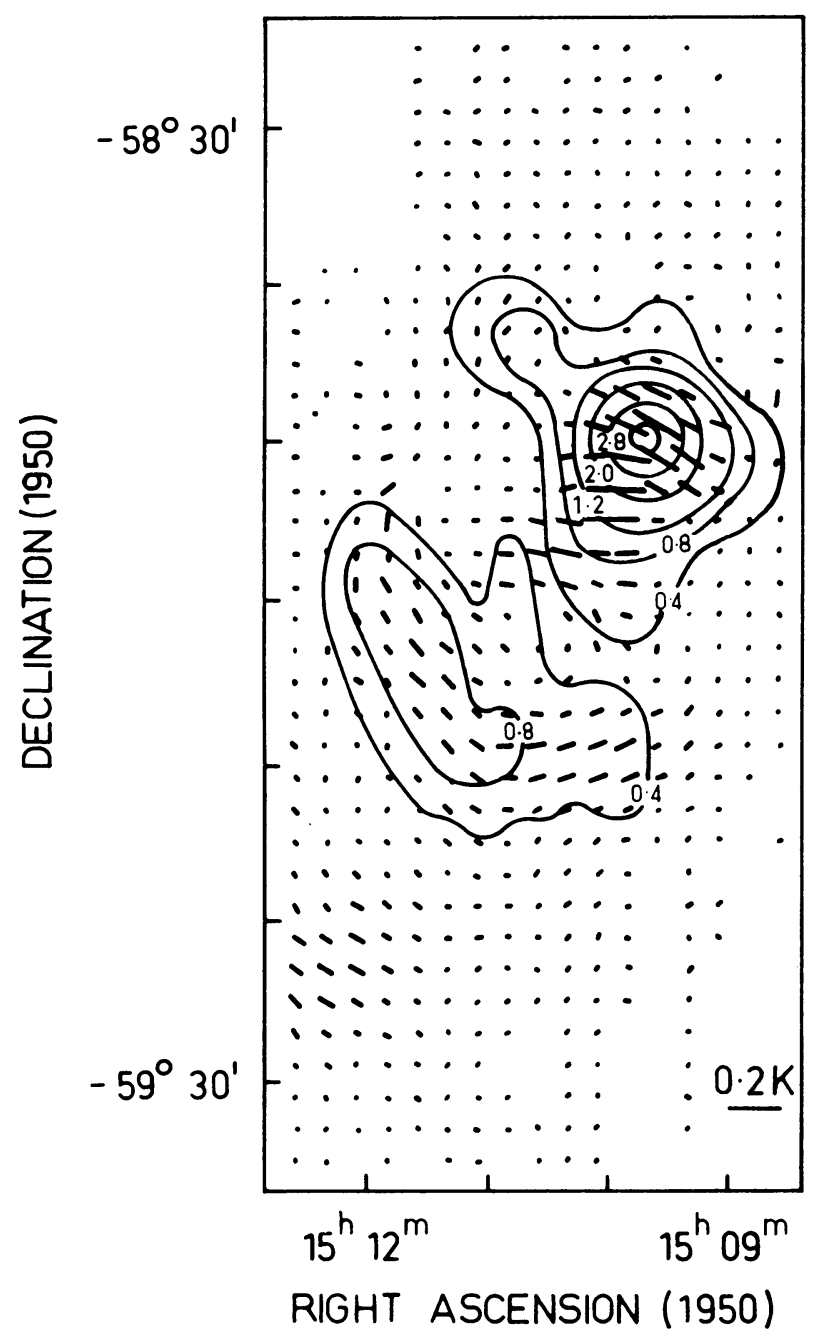

Fig. 4a. $5000-\mathrm{MHz}$ E vectors for MSH 15-52.

accepted. It is of course possible that additional rotations by multiples of $\pi$ radians could occur between $2700 \mathrm{MHz}$ and $5000 \mathrm{MHz}$, but each of these would change the rotation measure by $350 \mathrm{rad} \mathrm{m}^{-2}$ and in no case where a third frequency is available has this been observed within the errors imposed on the measured position angles. In the case of MSH 14-63 discussed above, the Faraday rotation was quite small and varied only very gradually across the source. This was confirmed by a relatively small depolarization ratio between the two frequencies.

Comparison of the $\mathbf{E}$ vectors at 6 and $11 \mathrm{~cm}$ for the extended Vela X SNR (G263.4-3.0) (Milne, 1973) shows again only a very small Faraday rotation. The average value was $+56 \mathrm{rad} \mathrm{m}^{-2}$ with an extreme range from $-100 \mathrm{rad} \mathrm{m}^{-2}$ to 


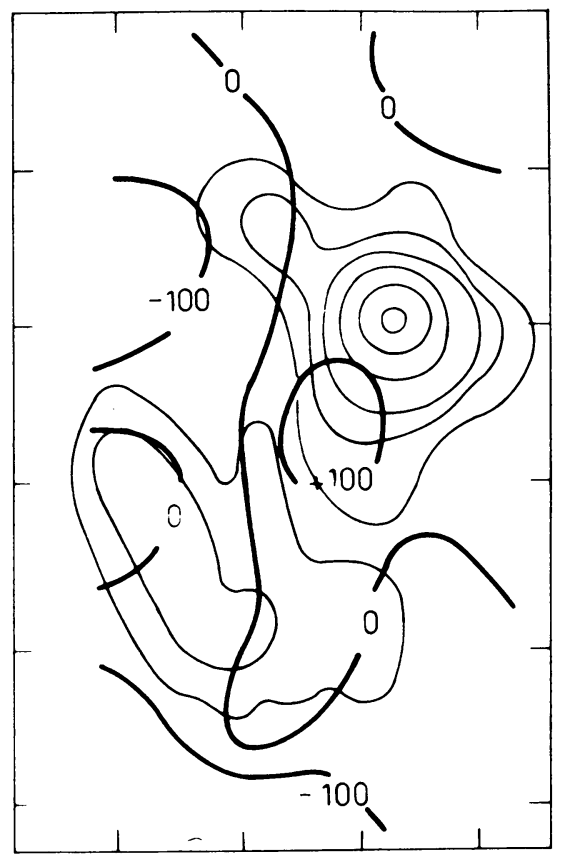

Fig. 4b. Distribution of rotation measure $\left(\mathrm{rad} \mathrm{m}^{-2}\right)$.

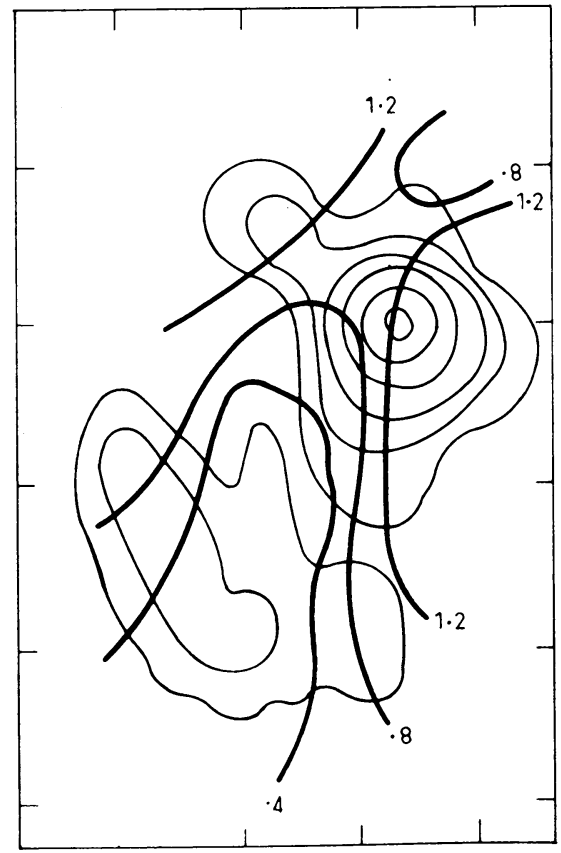

Fig. 4c. Depolarization ratio $\left(P_{2700 \mathrm{MHz}} / P_{5000 \mathrm{MHz}}\right)$. 


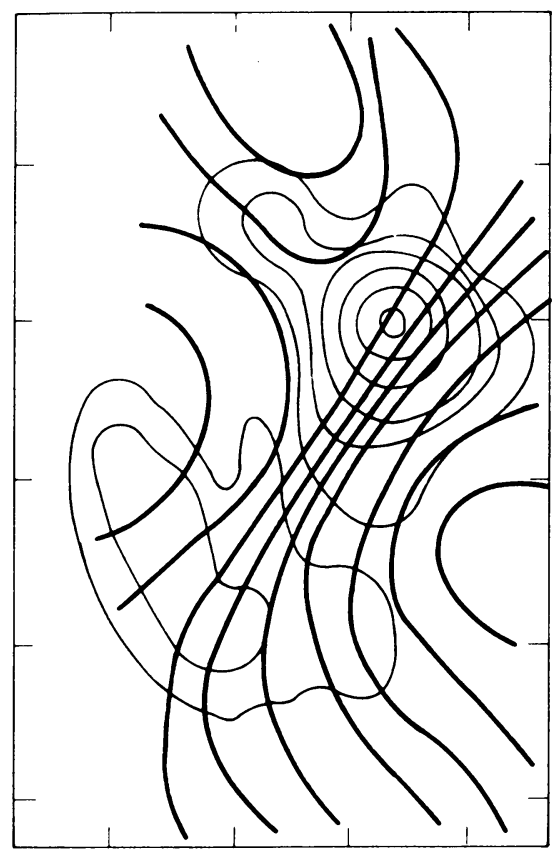

Fig. 4d. The directions of magnetic field.

$+150 \mathrm{rad} \mathrm{m}^{-2}$. This amount of rotation could reasonably be internal to the source since for a source with a diameter of $20 \mathrm{pc}$ and a longitudinal magnetic field strength of $10^{-5} \mathrm{G}$ a rotation measure of $56 \mathrm{rad} \mathrm{m}^{-2}$ implies a density of only $0.33 \mathrm{~cm}^{-3}$ for the thermal electrons in the medium. However it is equally acceptable that all of this rotation is between the object and the Sun. Another illustration of the small general Faraday rotation is given in Figures $4 a, b$, c, which show respectively the 6-cm $\mathbf{E}$ vectors, the Faraday rotation, and the depolarization ratio (per cent polarization at $11 \mathrm{~cm} /$ per cent at $6 \mathrm{~cm}$ ) for the source MSH 15-52 (G320.4-1.2). The small depolarization is, of course, consistent with only weak gradients in the Faraday rotation across the beam.

(c) MAGNETIC FIELD CONFIGURATION

After correcting for the Faraday rotation shown above, the orientations of the projected magnetic field lines for MSH 15-52 are as shown in Figure 4d. Although some areas show a more or less radial pattern the general structure is quite confused. A better example of a more regular field including a very significant radial component is shown in our results for Puppis A (G260.4-3.4) (Figures 5a, b, c and d, the $6 \mathrm{~cm} \mathbf{E}$ vectors, the Faraday rotation, depolarization ratio and the magnetic field orientations, respectively). We note in passing that, perhaps fortuitously, the position of most significant Faraday rotation northwest near the position of the extended X-ray 


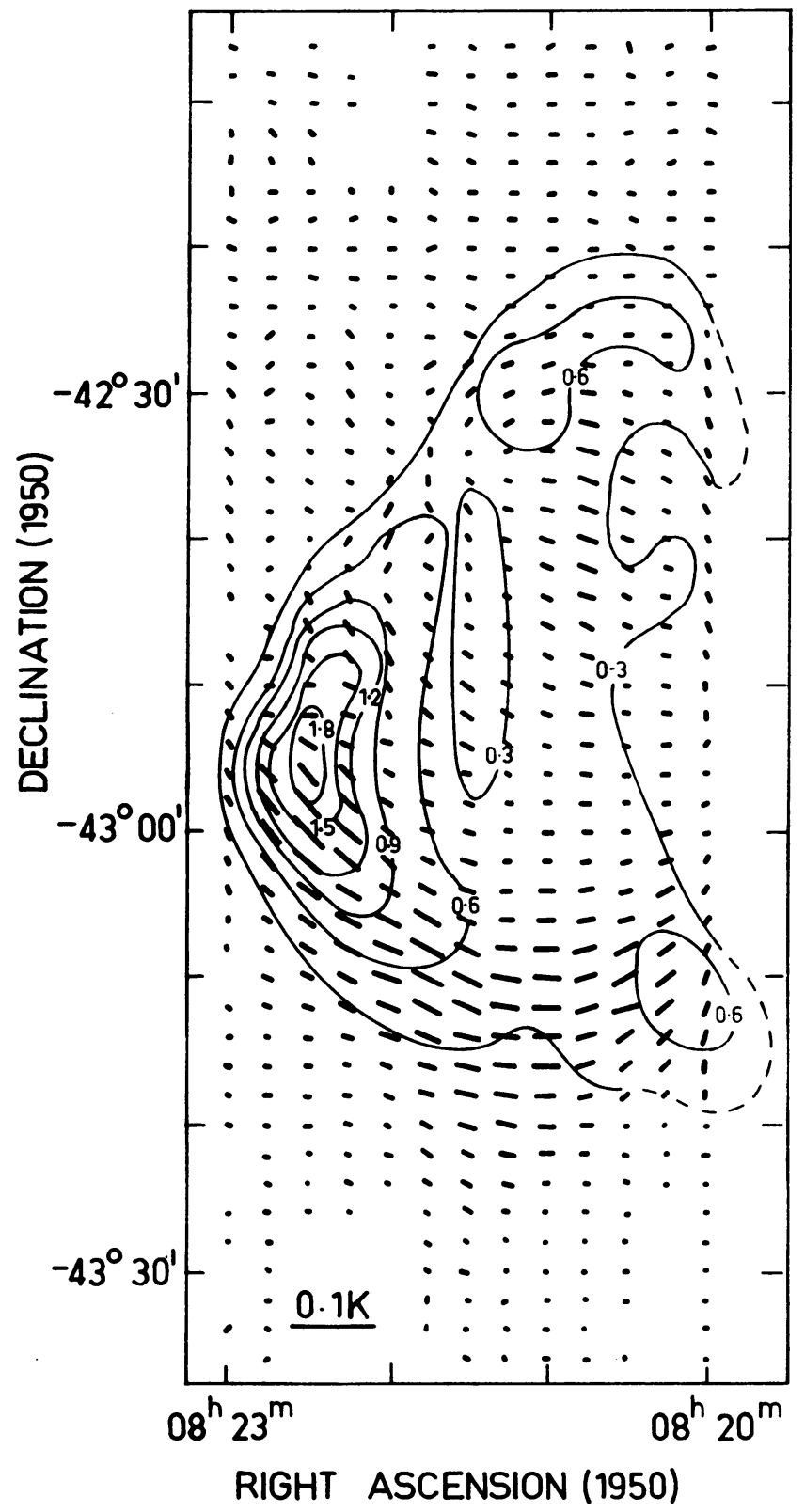

Figs. 5a-d. The same as Figure 4 for Puppis A. 

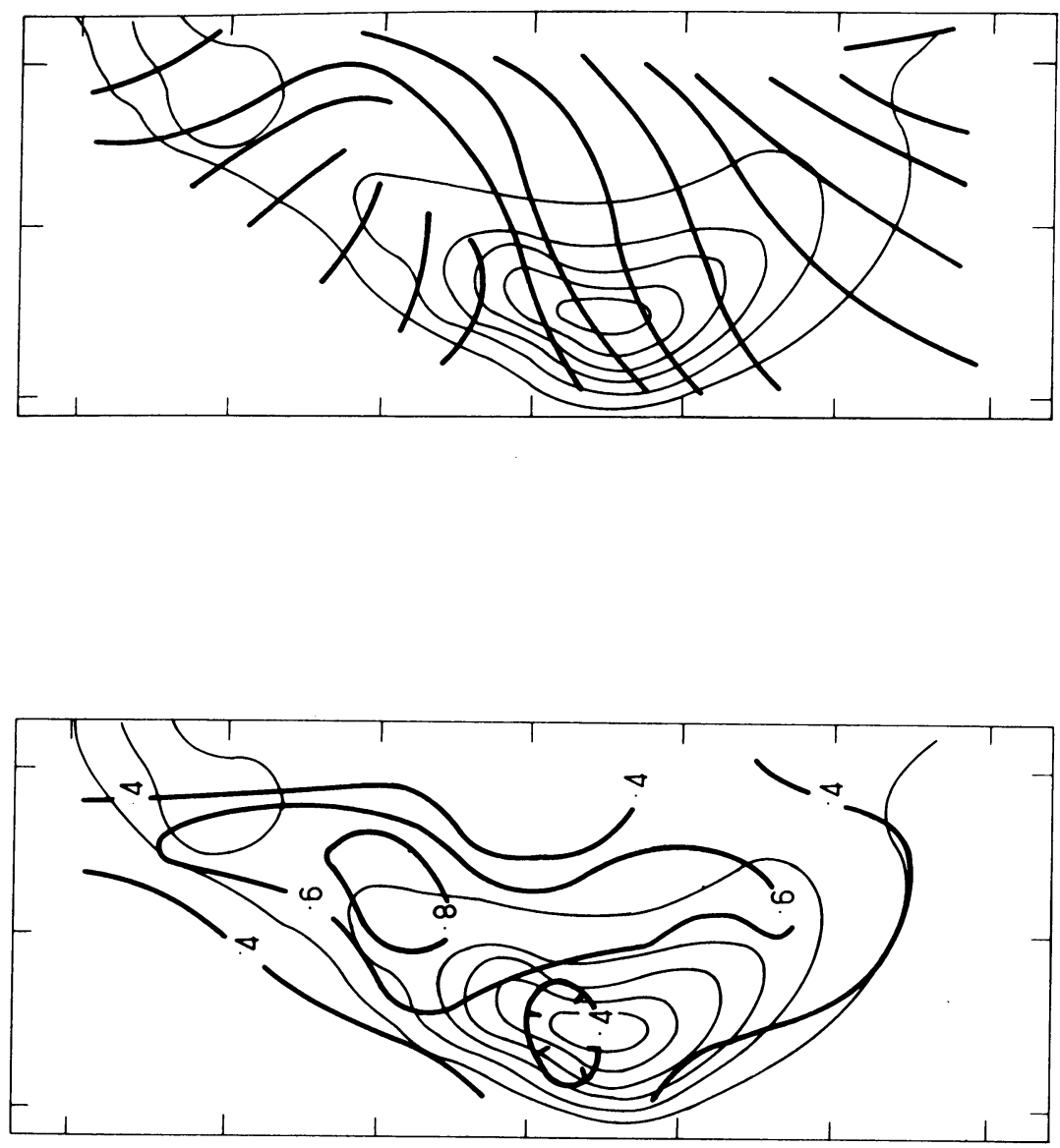

$\stackrel{\substack{n \\ i}}{i=0}$

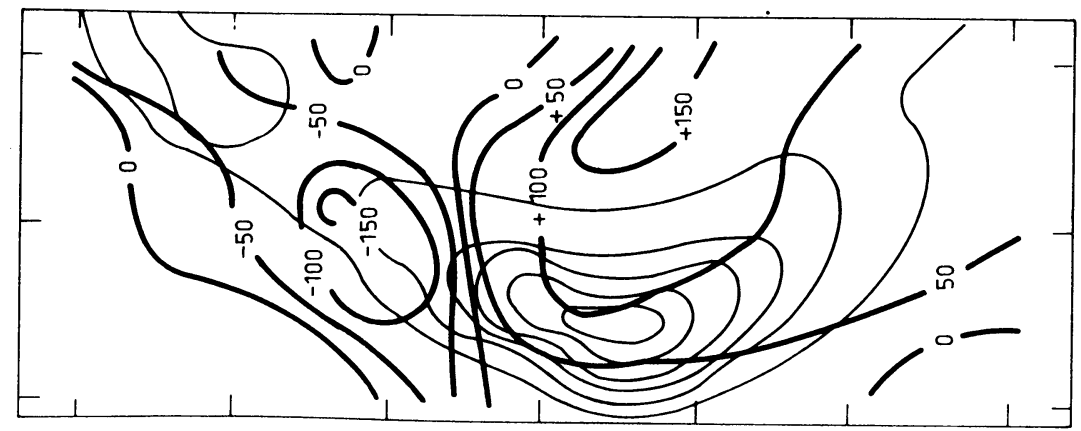

ค่ 
source associated with the SNR (Zarnecki et al., 1973). As a final figure (Figure 6) we show the magnetic field vectors of Vela $X$ as determined from the data shown above superimposed upon an optical photograph of the region. The vector lengths are the mean of the polarized intensities at 6 and $11 \mathrm{~cm}$, and the circle marks the position of the pulsar. Clearly there is polarization associated with the SNR, but its pattern including such features as the whorl in the south centre will be very difficult to evaluate even with three-dimensional models.

In addition to the results shown above we recall the tangential field found for $1209-51 / 52$ (G296.3 + 10.0) (Whiteoak and Gardner, 1968, confirmed by us at $6 \mathrm{~cm}$ ) and the nearly uniform field across W44 (G34.6-0.5) (Kundu and Velusamy, 1972; Milne and Dickel, 1973). Thus from this preliminary analysis of a few sources, no

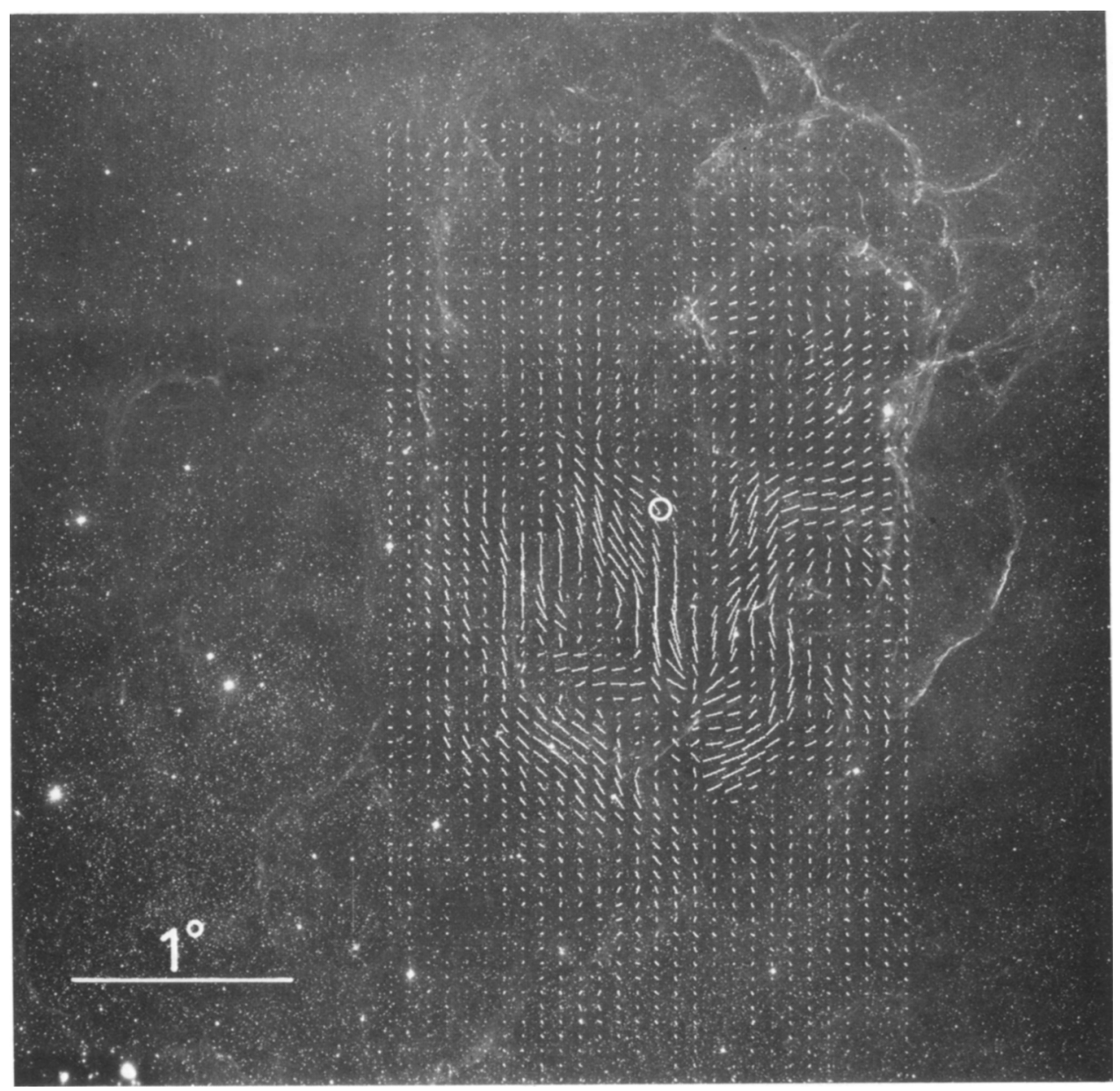

Fig. 6. Vela X showing the optical features (courtesy of B. J. Bok) overlaid with vectors representing the direction of the projected magnetic field. The length of these vectors is the average of the $5000-\mathrm{MHz}$ and $2700-\mathrm{MHz}$ polarization intensity. 
clear picture of the magnetic field configurations has emerged, and it would appear that the individual SNR had very different starting conditions and/or very varied interaction with the surrounding interstellar medium. If any general trend is seen it would be that the mean percentage polarization increases with decreasing surface brightness, i.e., the older remnants perhaps have more orderly fields.

\section{Conclusions}

In most directions along the galactic plane there is strong polarization of the background emission with a scale comparable with the angular size of many of the SNR. Studies of these objects should therefore include sufficient measurements of the background to assess its effect.

Working within the error in position angle we find that rotation measures generally lie within the range $\pm 200 \mathrm{rad} \mathrm{m}^{-2}$ and vary uniformly across the source. Except in very few objects the magnetic fields delineate patches with uniform direction but bearing no relation to the source structure.

\section{References}

Kundu, M. R. and Velusamy, T.: 1972, Astron. Astrophys. 20, 237.

Milne, D. K.: 1972, Australian J. Phys. 25, 307.

Milne, D. K.: 1973, The Vela Supernova Remnant, in preparation.

Milne, D. K. and Dickel, J. R.: 1973, submitted to Australian J. Phys.

Whiteoak, J. B. and Gardner, F. F.: 1968, Astrophys. J. 154, 807.

Zarnecki, J., Culhane, J. L., Fabian, A. C., Rapley, C. G., Silk, R., Parkinson, J. H., and Pounds, K. A.: 1973, Nature Phys. Sci. 243, 4.

D. K. Milne

Division of Radiophysics, CSIRO,

P.O. Box 76, Epping, N.S.W. 2121, Australia

John R. Dickel

University of Illinois Observatory, Urbana, Ill. 61801, U.S.A.

(Discussion follows after paper by R. M. Duin et al., p. 361.) 